\title{
An EUDET/AIDA Pixel Beam Telescope for Detector Development
}

\author{
Igor Rubinskiy ${ }^{1}$ \\ DESY \\ Notkestrasse 85, Germany \\ E-mail:igor.rubinskiy@desy.de

\section{Hanno Perrey} \\ DESY \\ Notkestrasse 85, Germany \\ E-mail: hanno.perrey@desy.de
}

\begin{abstract}
A beam telescope based on CMOS pixel sensors was developed within the EUDET project offering high track pointing resolution $(\sigma \sim 2 \mu \mathrm{m})$. The telescope consists of six CMOS sensor planes (Mimosa26) with a pixel pitch of $18.4 \mu \mathrm{m}$ and a thickness $50 \mu \mathrm{m}$. The excellent resolution, readout rate and DAQ integration capabilities made the telescope a primary test-beam tool for many groups including several CERN based experiments.

Within the European detector infrastructure project AIDA the test-beam telescope is being further extended in terms of cooling and powering infrastructure, read-out speed, area of acceptance, and precision. In order to provide a system optimized for different requirements set by the user community a combination of various state-of-the-art pixel technologies is foreseen. Furthermore, new central dead-time-free trigger logic unit (TLU) has been developed to provide LHC-speed response with one-trigger-per-particle operating mode and a synchronous clock for all connected devices. In this report, the design and current development status of this even more flexible telescope with three different pixel technologies (ATLAS FE-I4, Mimosa, TimePix) is presented.
\end{abstract}

Technology and Instrumentation in Particle Physics 2014

2-6 June, 2014

Amsterdam, the Netherlands 


\section{Introduction}

The technology development for High Energy Physics (HEP) detectors has motivated and equally profited from the development of the test-beam infrastructure facilities at DESY and CERN. In line with the European Strategy for Particle Physics the detector technology development is considered as one of the building blocks mandatory for the success of the HEP physics programs in Europe. In this paper we overview the test-beam tracking telescope developed within the European Union Framework Programs (EU FP) EUDET and AIDA, which took place in 2006-2010 and 2011-2014 respectively.

The EUDET project has been planned and carried out with the focus on the International Linear Collider (ILC) community and aimed at detector development towards the electronpositron collider physics at the ILC. One of the Joint Reasearch Activity groups (JRA1) has developed a, now worldwide known, EUDET telescope based on high-resolution and low material budget CMOS pixel technology sensors (Mimosa26). Its most distinctive features are the integration friendly interfaces provided by the Trigger Logic Unit (TLU), software DAQ framework (EUDAQ), and generic offline data reconstruction and analysis library, EUTelescope, which operates within the ILCSoft/Marlin framework. A detailed overview of the EUDET telescope can be found elsewhere [1].

The EUDET project has been continuied with EU FP7 AIDA with the goal to maintain and improve the existing facilities. The AIDA early achievements have been reported elsewhere [2]. The demand for the EUDET type telescope within EUDET and AIDA projects has been steadily increasing over the years (Figure 1).

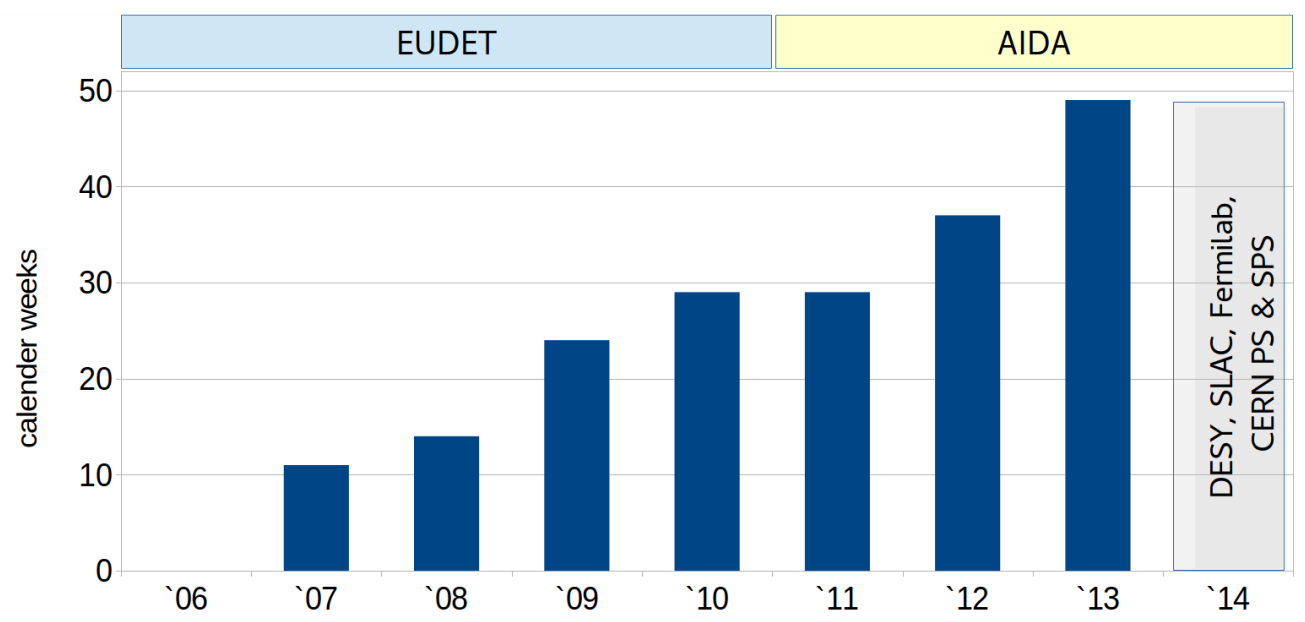

Figure 1. The number of calendar weeks for which a EUDET telescope was requested by detector R\&D groups. The EUDET demonstrator with analog Mimosa sensors was available from 2007 till 2009, when it was superseded by digital Mimosa26 sensors. Within the AIDA project several replicas of the telescope have been built by DESY to match the demand of the user groups at test-beam lines worldwide. In the year 2014 all high energy beam lines both in Europe and USA are hosting one of the telescope replicas.

\section{The EUDET telescope}

The pixel beam telescope designed within EUDET project features six CMOS pixel detectors (Mimosa26), each with $18.4 \mu \mathrm{m}$ square pixels arranged in 1152 columns and 576 rows. This 
gives in total about four million readout channels which makes it alone comparable to the large scale collider experiments at HERA and Fermilab which took place in the recent decades. The AMS $350 \mathrm{~nm}$ CMOS technology used for manufacturing of the Mimosa26 detectors is an industry standard used in a variety of commercial applications. The simple three transistor approach to collect diffused charge produced in the underlying $10 \mu \mathrm{m}$ epitaxial layer allows the construction of pixels with a pitch of less then $20 \mu \mathrm{m}$ and a sensitive thickness below $20 \mu \mathrm{m}$. With the rising industrial interest in high resistivity epitaxial silicon for CMOS detectors the production cost of improved noise and radiation hardness parameters became already possible with $\sim 400 \mathrm{Ohm} \cdot \mathrm{cm}$ for $10 \mu \mathrm{m}$ epitaxial layer for Mimosa26. The average noise occupancy does not exceed $10^{-5}$ for non-irradiated sensors at room temperature at the signal threshold level consistent with an average efficiency above $98 \%$. For stable performance the detectors are kept at $\sim 18 \mathrm{C}$ degree. The readout of the Mimosa26 detectors is performed in a rolling shutter approach, which takes 16 cycles of an $80 \mathrm{MHz}$ clock per row, with all 1152 columns being readout in parallel. The 16 clock cycles allow for a Correlated Double Sampling and Zero Suppression being performed on-chip with the digital circuitry placed outside of the pixel array. At this clock frequency the Mimosa26 integration time equals $115.2 \mu$ s and allows about 8680 frames per second to be read out. With the on-chip buffer size being able to accommodate the data of about a few hundred pixels (above threshold) on the sensor, the expected maxium rate of particles through the active area estimates to about $1 \mathrm{MHz}$ particles per $\mathrm{cm}^{2}$. Four Hamamatsu Photo Multiplier Tubes (PMT) assemblies with scintillators and lightguides, two in front and two in the back of the telescope, define the trigger window. The cross configuration of the scintillators with a size of $1 \mathrm{~cm}$ and $2 \mathrm{~cm}$ forms a rectangular window matching the Mimosa26 acceptance area. The powering of the PMTs can be provided by a two channel power supply and, alternatively, by a Trigger Logic unit (TLU), which provides the trigger decision and distribution of the trigger signal to all DAQ systems integrated with the telescope. The TLU, based on a commercial Spartan3 board, uses several custom-made add-on PCBs to interface PMT signals, and attached devices with RJ45, and LEMO (NIM or TTL) connectors. The software integration of the DAQ systems and the TLU, together with a run control GUI, logging, data storing, and online monitoring systems are provided within the EUDAQ framework. EUDAQ serves as toolset and an integration layer for DAQ systems, providing the communication protocols for them to participate in a common DAQ. The design of the EUDET TLU and EUDAQ explicetly requires each DAQ system to issue a data packet readout by its detector electronics, and send it to a central Data Collector. This scheme results in only one EUDAQ Event per every trigger signal issued by the TLU, with no subsequent triggers recorded until all DAQ systems indicate to the TLU their availability to accept new triggers. This architecture limits the overall trigger rate by the DAQ system with slowest readout cycle, which in practical applications leads to a maximum rate of few thousand particles per second. The variety of beam lines at CERN (PS, SPS), DESY, SLAC, Fermilab, brings also differences in the beam spill structure. This limits the average particle rate to below $1 \mathrm{kHz}$. The offline track reconstruction and data analysis is performed within ILCSoft/Marlin framework. The framework building blocks are organized as Processors, which can be configured in an XML steering file, where the order and parameters of the Processors can be adjusted. This way the Marlin/Processor architecture gives a lot of flexibility to the end user. The telescope processors are collected in the EUTelescope library and encapsulate all basic data reconstruction steps from channel (pixel) clustering to individual detector alignment, track reconstruction, and DUT analysis. The telescope performance has been validated at CERN (120 GeV pions) and 
DESY ( 1 to $6 \mathrm{GeV}$ positrons). In order to get realistic data description at DESY beam energies all scattering material between sensitive planes (Figure 2) has to be taken into account. The precision of the track prediction at the DUT (plane \#3) based on five other telescope planes is shown on Figure 3. The combination of the thinness of the telescope planes $(50 \mu \mathrm{m})$ with their hit position precision $(\sim 3.5 \mu \mathrm{m})$ when minimising the distances to the Detector Under Test (DUT), allows to sustain track pointing precision below $3 \mu \mathrm{m}$ for all electron (positron) energies above $2 \mathrm{GeV}$ and distance to the DUT shorter then $20 \mathrm{~mm}$ (Figure 3).

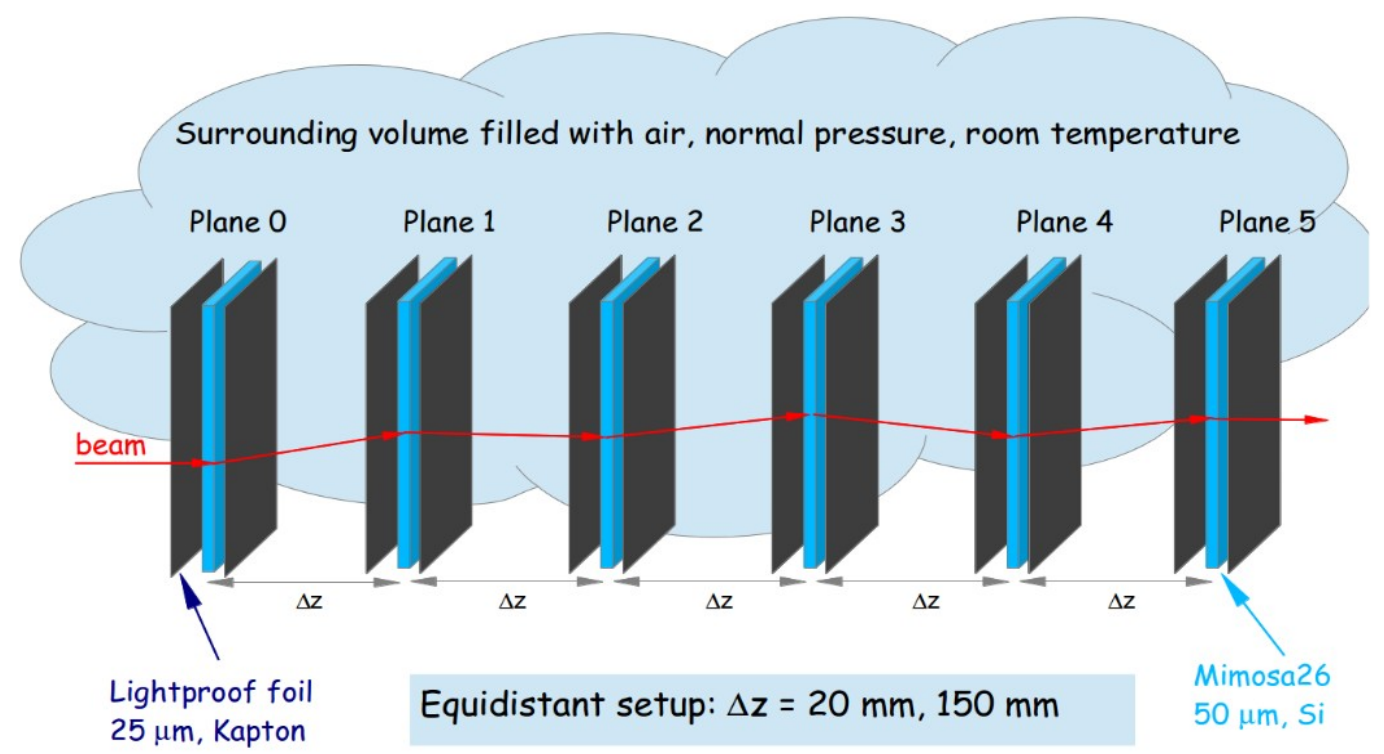

Figure 2: The scattering material around the telescope measurment plane. The planes are counted with zero starting upstream the beam direction.

\section{The demand for the test-beam telescopes}

The pixel beam telescope designed within EUDET and its continuing support both in EUDET and AIDA programs has gained a lot of acknowledgement by the community. The EUDET telescope replicas have been requested and installed not only at DESY as permanent location, but also purchased by the detector R\&D user groups, such as the ATLAS collaboration, Bonn University and Carlton University. Due to the long shut-down at CERN, where no testbeam lines were operating, the DESY test-beam facility has attracted many detector R\&D groups to perform their test-beam programs with replicas of the EUDET telescope installed at the three beam lines of the DESY II machine (Figure 4). During the 49 weeks of the DESY II running period in 2013 there were 123 beam weeks requested by the detector R\&D groups, of which two thirds were telescope users. 


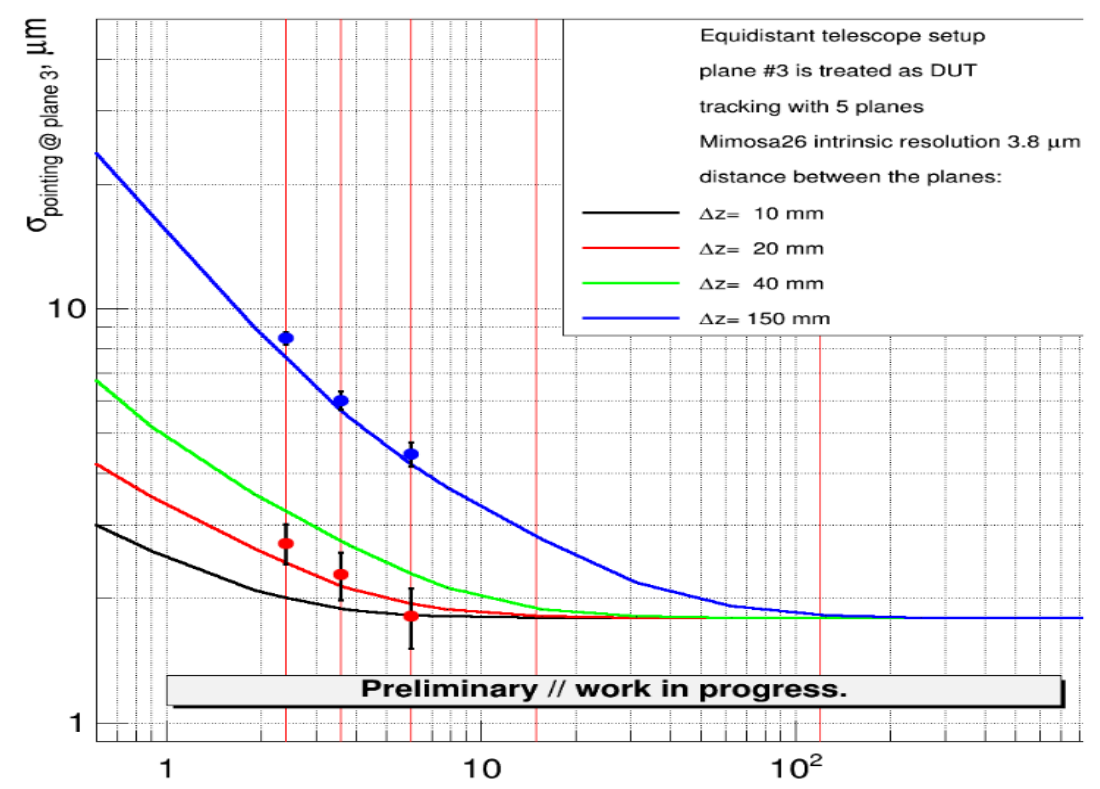

Figure 3: Telescope pointing resolution with five planes achived at plane 3 . The data points are deconvoluted using nominal intrinsic resolution of the plane $3.8 \mu \mathrm{m}$
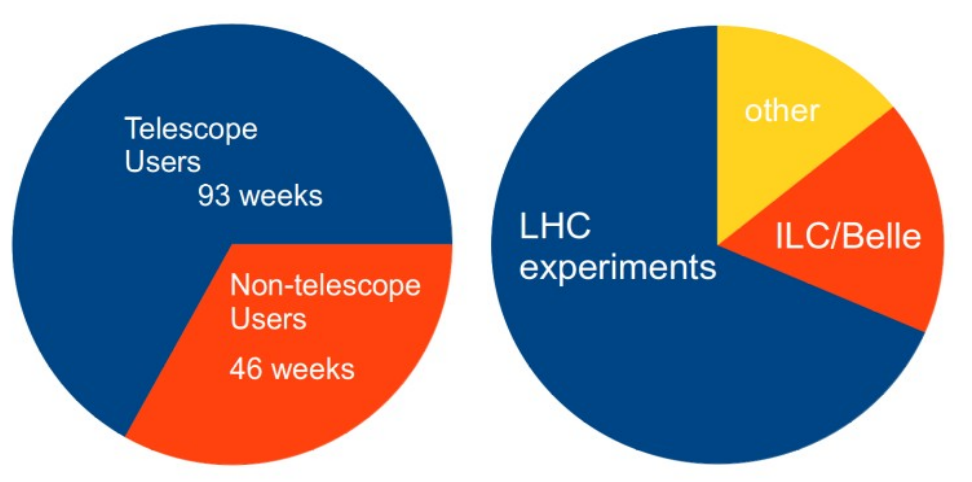

Figure 4: The DESY user weeks share in 2013 - early 2014. The lefthand plot (a) showing the share of the user groups request the telescope for their test-beams and on the righthand plot (b) the ratio of the LHC groups, lepton collider detector groups, and generic R\&D groups is shown.

\section{From EUDET to AIDA telescope}

The EUDET telescope featuring the flexible and generic Trigger-Busy handshake mechanism with the EUDET TLU and centralised data collection within EUDAQ framework has demonstrated also certain bottlenecks which have been reworked within the AIDA project. 


\subsection{Trigger system}

The Trigger Logic unit and the trigger distribution system are upgraded to a system with a no-handshake trigger and common clock distribution mechanism (Figure 5). The no-handshake trigger distribution removes the latencies imposed by the varying transmission lines length and the DUT DAQ feedback implementation. The AIDA TLU should allow much higher triggering rate reaching one million triggers per second, which is at least one order of magnitude higher comparing to the EUDET TLU.

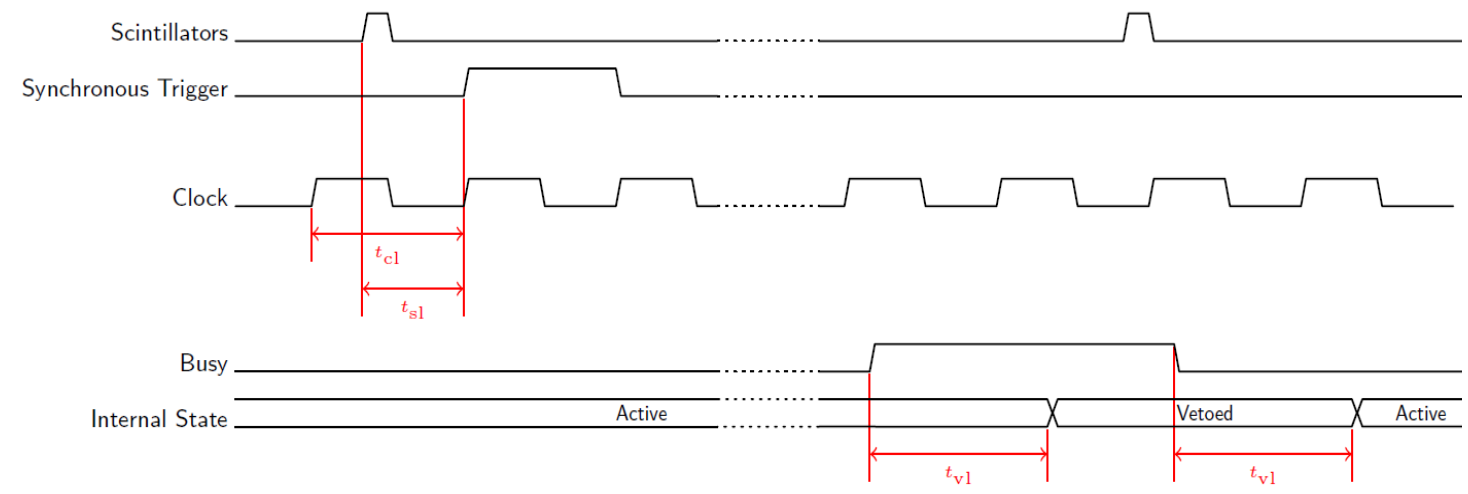

Figure 5: AIDA TLU synchronious trigger mode (from [3]).

\subsection{Integration of DAQ systems}

In the synchronous trigger distribution mode the DAQ systems participating in the test-beam, including the telescope's DAQ, become responsible for keeping record of the triggers accepted by the system and the clock cycle number associated to it. This approach requires the DAQ systems to write their own independent data streams to be merged back based on the list of trigger timestamps in the data streams arranged in data packets in contrary to the data events model in EUDAQ 1.0. Further details on the implementation design can be found in [4]. The first beam tests of the AIDA TLU and EUDAQ 2.0 are planned in the CERN PS operation in Summer 2014.

\subsection{Track time-stamping}

In the synchronous trigger distribution mode the DAQ systems participating in the test-beam are expected to accept and record a clock cycle number for every incoming trigger. In this operation mode some detector systems (like Mimosa26) can have up to a hundred particles in one readout packet, sharing one coarse granularity time-stamp, which have to be associated to a list of fine trigger timestamps from the TLU without space information of the particles. In order to solve the space-time matching problem a fast detector based on ATLAS FEI4 chip has been foreseen in the AIDA telescope. The FEI4 based module providing a flexible triggering capabilities [5] and in case it is included in the readout data stream also the time-stamping for every particle triggered by the TLU. The overall timing resolution below $1 \mathrm{~ns}$ can be obtaind from the AIDA TLU and the space pointing resolution coming from the six Mimosa26 planes, to be 
kept at about $2 \mu \mathrm{m}$. The Timepix chip family is considered as an alternative for the high rate test-beam telescope. The Timepix telescope built by the Medipix and LHCb collaboration has been provided access to within the AIDA Transnational Access and providing similar performance in terms of the average resolution and time resolution at high beam energies. The Timepix modules integration in the EUDET telescope (TLU handshake, EUDAQ, and EUTelescope reconstruction package) have been performed by CLICpix collaboration. This has demonstrated the possibilities to use same user interface at beam tests for detectors with continuous readout (Mimosa26), trigger based readout (FEI4), and shutter based system (Timepix).

\subsection{AIDA telescope software status}

The overall review of the AIDA telescope software development has been presented separately at this conference and is described elsewhere [4].

\section{Conclusions.}

In this paper we have discussed the EUDET/AIDA telescope work aiming at the upgrade of the telescope as test-beam infrastructure which will be able to provide high track pointing resolution at the wide energy range of the test-beam facilities at DESY, SLAC, CERN and Fermilab. Additional flexible triggering and time-stamping will allow to significantly extend the beam rate capabilities of the AIDA telescope, reaching up to one million tracks per second in the wide beam momentum range, also with a DUT DAQ system integrated into the telescope infrastructure.

\section{Acknowledgements}

The research leading to these results has received funding from the European Commission under the FP7 Research Infrastructures project AIDA, grant agreement no. 262025. The information herein only reflects the views of its authors and not those of the European Commission and no warranty expressed or implied is made with regard to such information or its use.

\section{References}

[1] I.Rubinskiy et al, An EUDET/AIDA Pixel Beam Telescope for Detector Development. Physics Procedia Volume 37, pp 1-2178 (2012)

[2] H.Perrey et al, An EUDET / AIDA Pixel Beam Telescope for Detector Development. 2012 IEEE NSS/MIC, N42-4

[3] F. Crescioli, D. Cussans, A. Dosil Suarez, AIDA-Note 2014, "AIDA mini-TLU manual"

[4] H.Perrey, I.Rubinskiy, TIPP2014 "EUDAQ and EUTelescope - Software Frameworks for Testbeam Data Acquisition and Analysis"

[5] T Obermann et al, 2014 JINST 9 C03035 doi:10.1088/1748-0221/9/03/C03035, Implementation of a Configurable FE-I4 Trigger Plane for the AIDA Telescope" 